\title{
Analysis of the Boundary Knot Method for 3D Helmholtz-Type Equation
}

\author{
F. Z. Wang ${ }^{1}$ and K. H. Zheng ${ }^{2}$ \\ ${ }^{1}$ School of Mathematical Sciences, Huaibei Normal University, Dongshan Road 100, Huaibei 235000, China \\ ${ }^{2}$ College of Water Conservancy and Ecological Engineering, Nanchang Institute of Technology, Tianxiang Road 289, \\ Nanchang 330099, China
}

Correspondence should be addressed to F. Z. Wang; wfz1984@gmail.com

Received 12 January 2014; Accepted 10 February 2014; Published 13 March 2014

Academic Editor: Yumin Cheng

Copyright (C) 2014 F. Z. Wang and K. H. Zheng. This is an open access article distributed under the Creative Commons Attribution License, which permits unrestricted use, distribution, and reproduction in any medium, provided the original work is properly cited.

Numerical solutions of the boundary knot method (BKM) always perform oscillatory convergence when using a large number of boundary points in solving the Helmholtz-type problems. The main reason for this phenomenon may contribute to the severely ill-conditioned full coefficient matrix. In order to obtain admissible stable convergence results, regularization techniques and the effective condition number are employed in the process of simulating 3D Helmholtz-type problems. Numerical results are tested for the 3D Helmholtz-type equation with noisy and non-noisy boundary conditions. It is shown that the BKM in combination with the regularization techniques is able to produce stable numerical solutions.

\section{Introduction}

During the past two decades, meshless methods have attracted great attention in the area of computational science and engineering. The boundary-type meshless methods, which used the boundary data only, is a hot topic in computational mechanics and computational mathematics. It can be divided into two types, that is, the week formulation based on boundary integral equations and the force collocation formulation. The first type is formed by combining the moving least-square approximation with boundary integral equation methods. Excellent researches include the boundary node method $[1,2]$, local boundary integral equation method (LBIE) [3-6], improved boundary node method [7-11], and boundary element-free method (BEFM) [12-15]. These methods have been successfully applied to many engineering problems; take the potential problem and elasticity problem as examples $[16,17]$. The second type includes the method of fundamental solutions (MFS) [18, 19], boundary knot method (BKM) [20], boundary collocation method [21], and modified method of fundamental solutions [22-26]. They are very popular in solving problems governed by homogeneous equations. The BKM and MFS are truly meshless since integration is no more needed as in the week formulation meshless method based on boundary integral equations. In particular, the BKM performs especially well for solving the Helmholtz-type problems [27, 28]. However, the convergence of the BKM as well as that the MFS is often unstable when the number of boundary points becomes large. The main reason of instability is largely due to the ill-conditioned full interpolation matrix [29-31].

As is known to all, regularization techniques are often used to deal with ill-conditioned cases encountered in inverse problems. For instance, a comprehensive comparison using the MFS and various regularization techniques have been made by Wei et al. in the study of inverse Cauchy problems [32]. The Tikhonov regularization (TR) method is used in the MFS solution of inverse source problems [33]. The truncated singular value decomposition (TSVD) is employed in the BKM solution of inverse problems [34, 35]. For direct problems, the singular value decomposition (SVD) is applied to resolve the ill-conditioning of the MFS [36]. However, Chen et al. [37] reexamined the results given in [36] and showed that there is no difference between using the direct Gaussian solver and the SVD for noise-free boundary conditions. They also demonstrated that the TSVD is clearly 
superior to Gaussian elimination for noisy boundary conditions. Recently, Wang et al. [29] have made a comprehensive study on regularization techniques in the BKM solution for direct problems in 2D cases.

For both direct and inverse problems, it is well known that the resulting matrices of the boundary-type meshless methods can be expressed in the standard form $\mathbf{A x}=$ b. Clearly, we should not rely solely on the $L^{2}$ condition number to predict accuracy of the computed solution of all practical ill-conditioned systems. Most importantly, the solution accuracy has an obvious dependence on the righthand side vector $\mathbf{b}$. So the effective condition number $(\mathrm{ECN})$, which is related to the right-hand side vector $\mathbf{b}$, is introduced to replace the $L^{2}$ condition number in the estimation of conditioning of the global interpolation methods [38-40]. In particular, the authors in [41] propose a new ECN, which will be used in this paper, to more accurately evaluate the conditioning of the resultant matrix in the solution process of the MFS.

It is the purpose of the present paper to provide the stability analysis of the 3D Helmholtz-type equations by using the BKM. To obtain a stable numerical scheme, various regularization techniques are employed for solving the resulting discretized system of equations. Then the relationship between the solution accuracy and the ECN of a linear system is investigated when using the BKM. Finally, we combine the BKM with various regularization techniques to examine Helmholtz problems under noisy and noise-free boundary conditions.

\section{The BKM}

We consider the time harmonic waves of frequency which leads to the $3 \mathrm{D}$ boundary value problems for the Helmholtztype equation

$$
\begin{gathered}
L u(\mathbf{x})=0, \quad \mathbf{x} \in \Omega, \\
u(\mathbf{x})=\bar{u}(\mathbf{x}), \quad \mathbf{x} \in \Gamma_{D}, \\
q(\mathbf{x})=\frac{\partial u(\mathbf{x})}{\partial n}=\bar{q}(\mathbf{x}), \quad \mathbf{x} \in \Gamma_{N},
\end{gathered}
$$

where $L=\Delta \pm \lambda^{2}, \Delta$ is the Laplace operator, $\lambda$ means the wave number, $\bar{u}=\bar{u}(\mathbf{x})$ and $\bar{q}=\bar{q}(\mathbf{x})(\mathbf{x}=(x, y, z))$ are the known functions, $\Omega$ denotes the $3 \mathrm{D}$ domain and $\partial \Omega\left(=\Gamma_{D} \cup\right.$ $\left.\Gamma_{N}\right)$ its boundary with Dirichlet boundary $\Gamma_{D}$ and Neumann boundary $\Gamma_{N}$, and $n$ represents the unit outward normal.

The nonsingular general solutions of the homogeneous Helmholtz operator $L=\Delta+\lambda^{2}$ and homogeneous modified Helmholtz operator $L=\Delta-\lambda^{2}$ are, respectively, given by

$$
\begin{gathered}
u^{*}(r)=\frac{\sin (\lambda r)}{r}, \\
u^{*}(r)=\frac{\sinh (\lambda r)}{r},
\end{gathered}
$$

with $r$ representing the Euclidean norm distance between two points $\mathbf{x}_{i}=\left(x_{i}, y_{i}, z_{i}\right)$ and $\mathbf{x}_{j}=\left(x_{j}, y_{j}, z_{j}\right)$. Since there is no singularity in nonsingular general solutions, all collocation and source points can be placed on the physical boundary simultaneously.

The basic theory of the BKM lies in that the solution of Helmholtz-type boundary value problems can be approximated by

$$
u\left(\mathbf{x}_{i}\right)=\sum_{j=1}^{N} \alpha_{j} u^{*}\left(r_{i j}\right), \quad \mathbf{x}_{i} \in \partial \Omega,
$$

where $N$ denotes the total number of boundary points and $\alpha_{j}$ is the unknown coefficients. By collocating (3) on Dirichlet and Neumann boundary conditions, we have

$$
\begin{gathered}
\sum_{j=1}^{N} \alpha_{j} u^{*}\left(r_{i j}\right)=\bar{u}\left(\mathbf{x}_{i}\right), \quad \mathbf{x}_{i} \in \Gamma_{D}, \\
\sum_{j=1}^{N} \alpha_{j} \frac{\partial u^{*}\left(r_{k j}\right)}{\partial n}=\bar{q}\left(\mathbf{x}_{k}\right), \quad \mathbf{x}_{k} \in \Gamma_{N} .
\end{gathered}
$$

Equation (4) can be written in the following matrix system:

$$
\mathbf{A} \alpha=\mathbf{b}
$$

where

A

$$
=\left(\begin{array}{cccc}
u^{*}\left(r_{1,1}\right) & u^{*}\left(r_{1,2}\right) & \cdots & u^{*}\left(r_{1, N}\right) \\
\cdots & \cdots & \cdots & \cdots \\
u^{*}\left(r_{N_{1}, 1}\right) & u^{*}\left(r_{N_{1}, 2}\right) & \cdots & u^{*}\left(r_{N_{1}, N}\right) \\
\frac{\partial u^{*}\left(r_{N_{1}+1,1}\right)}{\partial n} & \frac{\partial u^{*}\left(r_{N_{1}+1,2}\right)}{\partial n} & \cdots & \frac{\partial u^{*}\left(r_{N_{1}+1, N}\right)}{\partial n} \\
\cdots & \cdots & \cdots & \cdots \\
\frac{\partial u^{*}\left(r_{N, 1}\right)}{\partial n} & \frac{\partial u^{*}\left(r_{N, 2}\right)}{\partial n} & \cdots & \frac{\partial u^{*}\left(r_{N, N}\right)}{\partial n}
\end{array}\right)
$$

is an $N \times N$ interpolation matrix, $N_{1}$ is the number of points on Dirichlet boundary $\Gamma_{D}$ and the rest on Neumann boundary $\Gamma_{N}, \alpha=\left[\alpha_{1}, \alpha_{2}, \ldots, \alpha_{N}\right]^{T}$ is the unknown coefficients, and $\mathbf{b}=\left[\bar{u}_{1}, \ldots, \bar{u}_{N_{1}}, \bar{q}_{N_{1}+1}, \ldots, \ldots, \bar{q}_{N}\right]^{T}$ is the known boundary data. We notice that the BKM produces a highly illconditioned and dense matrix system when a large number of boundary points are used. For more details, we refer readers to $[29,30]$.

\section{Measurement of the Coefficient Matrix Conditioning}

The interpolation matrix $\mathbf{A}$ in (5) can be decomposed by the singular value decomposition (SVD)

$$
\mathbf{A}=\mathbf{U} \Sigma \mathbf{V}^{T}
$$

where $\mathbf{U}=\left[u_{1}, u_{2}, \ldots, u_{N}\right]$ and $\mathbf{V}=\left[v_{1}, v_{2}, \ldots, v_{N}\right]$ are orthogonal matrices, $\mathbf{U}^{T} \mathbf{U}=\mathbf{V}^{T} \mathbf{V}=\mathbf{I}_{N}$, where $\mathbf{I}_{N}$ denotes 
the identity matrix and $\Sigma$ is a diagonal matrix with diagonal elements $\sigma_{1} \geq \sigma_{2} \geq \cdots \geq \sigma_{N}>0$, where $\sigma_{i}, 1 \leq i \leq N$ are called singular values of $\mathbf{A}$. The $L^{2}$ condition number of a nonsingular square matrix $\mathbf{A}$ is defined by $\operatorname{Cond}(\mathbf{A})=$ $\|\mathbf{A}\| \cdot\left\|\mathbf{A}^{-1}\right\|$. Throughout this paper, the $L^{2}$ condition number can be stated as $\operatorname{Cond}(\mathbf{A})=\sigma_{1} / \sigma_{N}$.

Substituting (7) into (5), we have

$$
\alpha=\sum_{i=1}^{N} \frac{u_{i}^{T} \mathbf{b}}{\sigma_{i}} v_{i}
$$

For practical applications, the boundary data $\mathbf{b}$ may be disturbed by some noise. Clearly, we should not rely solely on the $L^{2}$ condition number to predict accuracy of the computed solution of all practical ill-conditioned BKM systems since the right-hand side vector $\mathbf{b}$ is excluded, particularly for the noisy boundary conditions. In many applications, $\mathbf{b}$ is problem-dependent but fixed. Under these conditions, as an alternative tool to estimate the accuracy of the BKM, we consider the effective condition number $\operatorname{ECN}=\operatorname{ECN}(\mathbf{A}, \mathbf{b})$ which is described in $[27,41]$ as

$$
\operatorname{ECN}(\mathbf{A}, \mathbf{b})=\frac{\|\mathbf{b}\|}{\sigma_{N} \sqrt{\left(\xi_{1} / \sigma_{1}\right)^{2}+\cdots+\left(\xi_{N} / \sigma_{N}\right)^{2}}},
$$

where $\xi_{i}$ depends on the right-hand side vector $\mathbf{b}$. For more details about other types of ECN, we refer readers to [39, 40, 42].

\section{Regularization Methods}

Based on the SVD, we briefly present three commonly used regularization techniques under two parameter choices, that is, the TSVD, the TR, and the damped singular value decomposition (DSVD) under parameter choice of the $L$ curve criterion (LC) and the GCV [43].

\subsection{Regularization Techniques}

TSVD. The fundamental theory of the TSVD lies in that the TSVD solution is often used to obtain a better estimate of the least squares solution. It is given by approximating a rank- $N$ full matrix $\mathbf{A}$ by a rank $K$ matrix in which only the largest $K$ singular values are retained

$$
\mathbf{A}_{K}=\sum_{i=1}^{K} u_{i} \sigma_{i} v_{i}^{T}
$$

In this instance, we can replace matrix $\mathbf{A}$ in (5) by $\mathbf{A}_{K}$, which has a well defined null space of dimension $N-K$ spanned by the right singular value vectors, $v_{K+1}, \ldots, v_{N}$. The original linear system (5) is then replaced by the following problem set of (11), where $\mathbf{b}$ is ideal noise-free data obtained at the minimized point. The resulting TSVD solution of

$$
\min \|\alpha\|_{2} \text { is subject to } \min \left\|\mathbf{A}_{K} \alpha-\mathbf{b}\right\|_{2}=\min
$$

is given by

$$
\alpha_{K}=\sum_{i=1}^{K} \frac{u_{i}^{T} \mathbf{b}}{\sigma_{i}} v_{i}
$$

where $K \leq N$ is a regularization parameter.

$T R$. The TR is a direct method in that the TR replaces the linear system (5) by the minimization problem

$$
\min _{\alpha \in R^{n}}\|\mathbf{A} \alpha-\mathbf{b}\|^{2}+\mu^{2}\|\alpha\|^{2}
$$

where $\mu \geq 0$ is a regularization parameter.

Based on the SVD, we can express (13) in terms of

$$
\alpha_{\mu}=\alpha_{\min }=\sum_{i=1}^{l} f_{i} \frac{u_{i}^{T} \mathbf{b}}{\sigma_{i}} v_{i},
$$

where $l$ is the rank of matrix $\mathbf{A}$ and

$$
f_{i}=\frac{\sigma_{i}^{2}}{\left(\sigma_{i}^{2}+\mu^{2}\right)}
$$

are the Wiener weights.

DSVD. A relatively less known regularization technique which is based on the SVD is the DSVD. Here, instead of using the filter factors in the TR, one introduces a smoother cut-off by means of filter factors $f_{i}$ defined as

$$
f_{i}=\frac{\sigma_{i}}{\sigma_{i}+\mu}
$$

and these filter factors decay slower than the TR filter factors and thus, in a sense, introduces less filtering.

The suitable value of the regularization parameter $\mu \geq 0$ is chosen by the $\mathrm{LC}$ or the GCV in this paper.

4.2. Regularization Parameters. The appropriate value choice for the regularization parameter $\mu$ remains an open problem till now [29]. Here, we briefly introduce the LC criterion and the GCV parameter choice $[44,45]$.

LC. The LC, which is considered as the most convenient graphical tool for analysis of discrete ill-posed problems, is defined as

$$
L:=\left\{\left(\log \left\|\alpha_{\mu}\right\|, \log \left\|\mathbf{A} \alpha_{\mu}-\mathbf{b}\right\|\right): \mu \geq 0\right\} .
$$

Note here that the $L$-curve is a continuous curve when the regularization parameter is real in the TR and the DSVD. In numerical computation, the point with maximum curvature will be searched as the corner of the $L$-curve. For the regularization methods with a discrete regularization parameter, such as in the TSVD, a finite set of points

$$
\left\{\left(\log \left\|\alpha_{q}\right\|, \log \left\|\mathbf{A} \alpha_{q}-\mathbf{b}\right\|\right): q=1,2, \ldots, N\right\}
$$

will be obtained and interpolated by a spline curve. The point on the spline curve with the maximum curvature is then chosen as the desirable regularization parameter. 

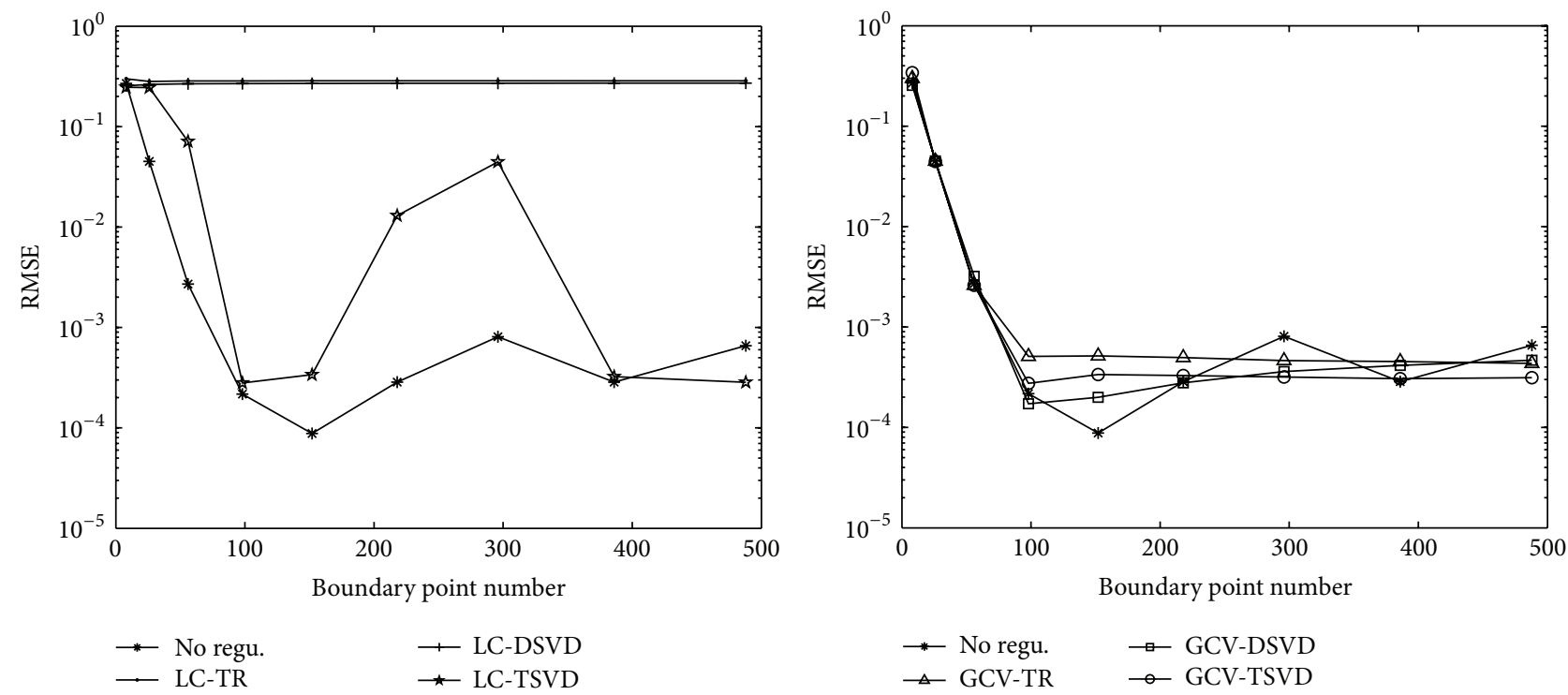

FIGURE 1: Root mean square errors for case 1.

The distinctive feature of the LC lies in that the regularized solution varies with the regularization parameter $\mu$.

$G C V$. To obtain the optimal value of the regularization parameter, the GCV is used to minimize the functional

$$
V(K)=\frac{(1 / N)\|(\mathbf{I}-\mathbf{A}(K)) \mathbf{b}\|^{2}}{[(1 / N) \operatorname{trace}(\mathbf{I}-\mathbf{A}(K))]^{2}},
$$

where $\mathbf{A}(K)$ is defined as

$$
\mathbf{A} \alpha_{K}=\mathbf{A}(K) \mathbf{b}
$$

The statistical method GCV is a predictive mean-square error criteria, in the sense that it estimates the minimizer of residual function

$$
T(K)=\frac{\left\|\mathbf{A}\left(\alpha_{K}-\alpha\right)\right\|^{2}}{N} .
$$

We note that $T$ is defined for both continuous and discrete regularization parameters.

\section{Numerical Examples}

In order to compare with the BKM with no regularization techniques, numerical results are given by using six regularization methods, that is, GCV-TR, LC-TR, GCV-DSVD, LCDSVD, GCV-TSVD, and LC-TSVD. by

Some random noise to the boundary conditions is added

$$
u=\bar{u}+\delta
$$

where $\delta=\varepsilon \times$ Rand [37]. Here, $\varepsilon$ denotes the noise level and the random number generator "Rand" is used to produce random numbers in $[-1,1]$. The root mean square error (RMSE) is used in the following cases [46].
5.1. Helmholtz Case on a Unit Cubic Domain. Here, we consider the $3 \mathrm{D}$ Helmholtz equation

$$
\Delta u+\lambda^{2} u=0, \quad(x, y, z) \in \Omega
$$

under boundary condition $u=\sin (x) \cos (y) \cos (z),(x$, $y, z) \in \partial \Omega$. The corresponding wave number is $\lambda=\sqrt{3}$.

Figure 1 displays the RMSE versus the boundary point number on a unit cubic domain $\Omega=\{(x, y, z) \mid 0<$ $x, y, z<1\}$. From which we notice that convergence curve of the BKM without regularization techniques, No regu., is oscillatory when the boundary point number becomes large. This phenomenon contradicts with the traditional opinion: a more accurate fitting of the exact data always leads to a better numerical results [47]. The reason may contribute to the highly ill-conditioned interpolation matrix $\left(\approx 10^{20}\right)$ which is shown in Figure 2 . Besides, we note that the effective condition number, ECN, is almost half the size of the condition number.

Using TSVD, DSVD, or TR under the regularization parameter LC, the accuracy of BKM seems to be even worse. On the other hand, the regularization techniques TSVD, DSVD, and TR under the regularization parameter GCV obtain more stable results. After the boundary point number $N>100$, the convergence curves have no oscillatory phenomenon. The GCV-TR method performs the best in this case. This is similar to the $2 \mathrm{D}$ cases [29].

Once noise is added on the boundary, it is seen from Table 1 that the ECN decreases while the RMSE increases using boundary point number $N=152$. We observe a sharp drop in the ECN as a tiny amount of noise $\varepsilon=1.0 \times 10^{-5}$ is added, which leads to a poor accuracy with RMSE = $1.14 \times 10^{0}$. This means that condition of the interpolation system in this case is worse than the 2D cases [27]. Even though all runs in Table 1 have exactly the same condition number, completely different errors and ECNs are observed in cases with higher noise levels. At the same time, the drop of 
TABLE 1: Boundary point number $N=152$ for case 1 .

\begin{tabular}{lccccc}
\hline Noise level & 0.0 & $1 \times 10^{-8}$ & $1 \times 10^{-7}$ & $1 \times 10^{-6}$ & $1 \times 10^{-5}$ \\
\hline Cond & $2.39 \times 10^{18}$ & $2.39 \times 10^{18}$ & $2.39 \times 10^{18}$ & $2.39 \times 10^{18}$ & $9.90 \times 10^{5}$ \\
ECN & $1.38 \times 10^{9}$ & $9.60 \times 10^{7}$ & $9.87 \times 10^{6}$ & $1.14 \times 10^{-1}$ & $9.90 \times 10^{4}$ \\
RMSE & $8.81 \times 10^{-5}$ & $1.20 \times 10^{-3}$ & $1.15 \times 10^{-2}$ & $2.86 \times 10^{-1}$ & $1.14 \times 10^{0}$ \\
LC-TR & $2.86 \times 10^{-1}$ & $2.86 \times 10^{-1}$ & $2.54 \times 10^{-4}$ & $2.69 \times 10^{-1}$ & $6.40 \times 10^{-3}$ \\
LC-DSVD & $2.69 \times 10^{-1}$ & $2.69 \times 10^{-1}$ & $6.51 \times 10^{-4}$ & $2.80 \times 10^{-3}$ & $7.24 \times 10^{-2}$ \\
LC-TSVD & $3.38 \times 10^{-4}$ & $4.51 \times 10^{-4}$ & $8.00 \times 10^{-3}$ & $9.00 \times 10^{-3}$ & $9.70 \times 10^{-3}$ \\
GCV-TR & $5.16 \times 10^{-4}$ & $4.96 \times 10^{-4}$ & $2.54 \times 10^{-4}$ & $8.60 \times 10^{-3}$ & $1.02 \times 10^{-2}$ \\
GCV-DSVD & $1.99 \times 10^{-4}$ & $2.27 \times 10^{-4}$ & $4.82 \times 10^{-4}$ & $8.50 \times 10^{-3}$ & $1.24 \times 10^{-2}$ \\
GCV-TSVD & $3.37 \times 10^{-4}$ & $1.76 \times 10^{-4}$ & $3.23 \times 10^{-4}$ & &
\end{tabular}

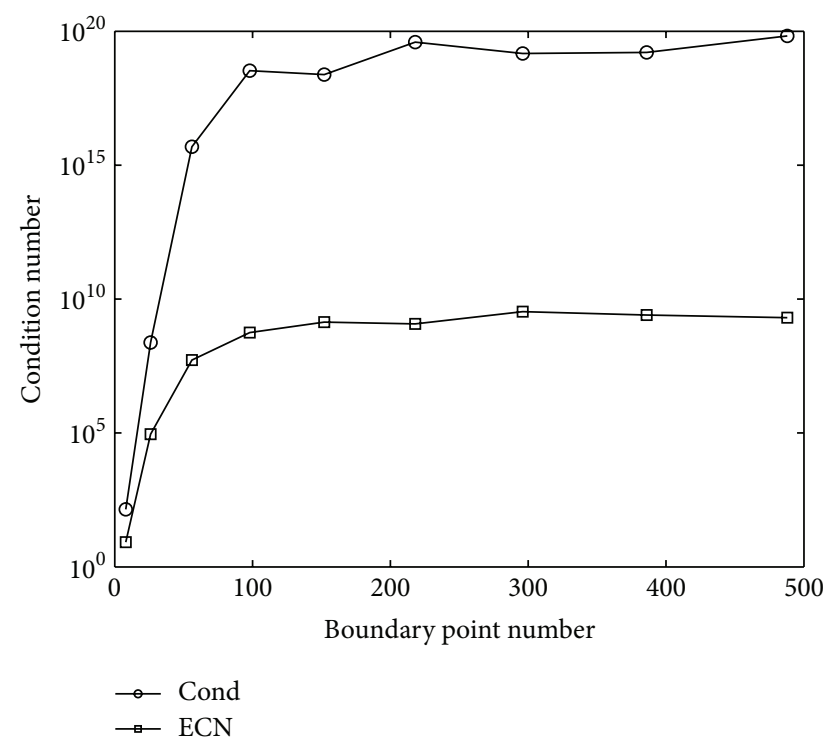

FIgURE 2: Condition number and ECN for case 1.

ECN has the same rate with the increase of the RMSE. More specifically, one order drop of the ECN corresponds with one order increase of the RMSE. This means that the ECN is a superior choice to the condition number for problems with noisy boundary conditions.

From Table 1, we observe that the relation between ECN and $\mathrm{RMSE}\left(\mathrm{ECN}=\mathrm{O}\left(\mathrm{RMSE}^{-1}\right)\right)$ is more strict than the relation observed in 2D spaces [27]. As more noise is added, the ECN is small enough (ECN $\left.<10^{5}\right)$ to indicate that the BKM solution (RMSE $=1.14 \times 10^{0}$ ) will not be accurate enough. This example is a good indication of the relationship between the accuracy of the BKM and the ECN because only the right-hand vector $b$ is altered with all other factors, including the ill-conditioned matrix $A$, staying constant.

For different fixed noise levels, we notice that the GCV based regularization techniques and LC-TSVD perform better than the other cases. Among which the GCV-TR and LC-TSVD give better solution accuracy with two-decimal precision than the one without regularization techniques. However, the other two LC based regularization techniques do not perform well. We point that the GCV base regularization techniques are less accurate with one-decimal precision than the one without regularization.

5.2. Helmholtz Case on a Unit Spherical Domain. In order to see the effect of the physical domain on solutions, we consider the 3D Helmholtz equation on a unit spherical domain $\Omega=$ $\left\{(x, y, z) \mid x^{2}+y^{2}+z^{2}<1\right\}$. Consider the following:

$$
\Delta u+\lambda^{2} u=0, \quad(x, y, z) \in \Omega
$$

under boundary condition $u=\sin (x)+\sin (y)+\sin (z)$, $(x, y, z) \in \partial \Omega$, with corresponding wave number $\lambda=1$.

For noise added on the boundary data, we observe from Table 2 that the ECN decreases while the RMSE increases for fixed boundary point number $N=144$. Obviously, the condition number is irrelevant with the noisy data. Although the condition number in case 1 is larger than the one in this case, the ECN in case 1 is smaller than the one in this case. This phenomenon again proves that the condition number has no relation to the ECN. More interestingly, we find that the ECN almost remains the same with nonnoisy case as a tiny amount of noise $\varepsilon=1.0 \times 10^{-8}$ is added. The corresponding solution accuracy RMSE $=5.77 \times 10^{-6}$ is similar with the nonnoisy case $\mathrm{RMSE}=3.24 \times 10^{-6}$. This means that condition of the interpolation system in this case is better than the previous case (Table 1 ). The main reason for the difference between case 1 and case 2 may be contributed to two reasons, that is, the different physical domain and the boundary value problems.

A sharp drop in the ECN is find as the noise $\varepsilon=1.0 \times 10^{-3}$ is added, which leads to solution accuracy with RMSE = $6.67 \times 10^{-1}$. As more noise is added, the solution accuracy gets even worse. Once regularization techniques are introduced, we find that the GCV based regularization techniques have better results than the LC based regularization techniques. This is similar to case 1 (Figure 1). If there is no noise added on the boundary, the GCV based regularization techniques have much more accurate solutions ( $\mathrm{RMSE} \approx 10^{-9}$ ) than the one without regularization techniques $\left(\mathrm{RMSE} \approx 10^{-6}\right)$. 
TABLE 2: Boundary point number $N=144$ for case 2 .

\begin{tabular}{|c|c|c|c|c|c|}
\hline Noise level & 0.0 & $1 \times 10^{-8}$ & $1 \times 10^{-3}$ & $1 \times 10^{-2}$ & $1 \times 10^{-1}$ \\
\hline Cond & $4.70 \times 10^{19}$ & $4.70 \times 10^{19}$ & $4.70 \times 10^{19}$ & $4.70 \times 10^{19}$ & $4.70 \times 10^{19}$ \\
\hline $\mathrm{ECN}$ & $5.32 \times 10^{8}$ & $1.90 \times 10^{8}$ & $1.61 \times 10^{3}$ & $1.62 \times 10^{2}$ & $1.97 \times 10^{1}$ \\
\hline RMSE & $3.24 \times 10^{-6}$ & $5.77 \times 10^{-6}$ & $6.67 \times 10^{-1}$ & $3.68 \times 10^{0}$ & $6.62 \times 10^{1}$ \\
\hline LC-TR & $8.06 \times 10^{-5}$ & $8.06 \times 10^{-5}$ & $7.00 \times 10^{-3}$ & $1.09 \times 10^{-2}$ & $6.20 \times 10^{-2}$ \\
\hline LC-DSVD & $3.25 \times 10^{-5}$ & $3.25 \times 10^{-5}$ & $6.20 \times 10^{-3}$ & $1.03 \times 10^{-2}$ & $6.40 \times 10^{-2}$ \\
\hline LC-TSVD & $2.75 \times 10^{-8}$ & $1.12 \times 10^{-7}$ & $1.00 \times 10^{-3}$ & $1.10 \times 10^{-2}$ & $1.02 \times 10^{-1}$ \\
\hline GCV-TR & $1.55 \times 10^{-9}$ & $9.86 \times 10^{-9}$ & $9.14 \times 10^{-4}$ & $9.30 \times 10^{-3}$ & $6.67 \times 10^{-2}$ \\
\hline GCV-DSVD & $5.58 \times 10^{-9}$ & $1.28 \times 10^{-8}$ & $9.85 \times 10^{-4}$ & $9.40 \times 10^{-3}$ & $7.16 \times 10^{-2}$ \\
\hline GCV-TSVD & $6.99 \times 10^{-9}$ & $9.52 \times 10^{-9}$ & $8.99 \times 10^{-4}$ & $9.10 \times 10^{-3}$ & $6.66 \times 10^{-2}$ \\
\hline
\end{tabular}
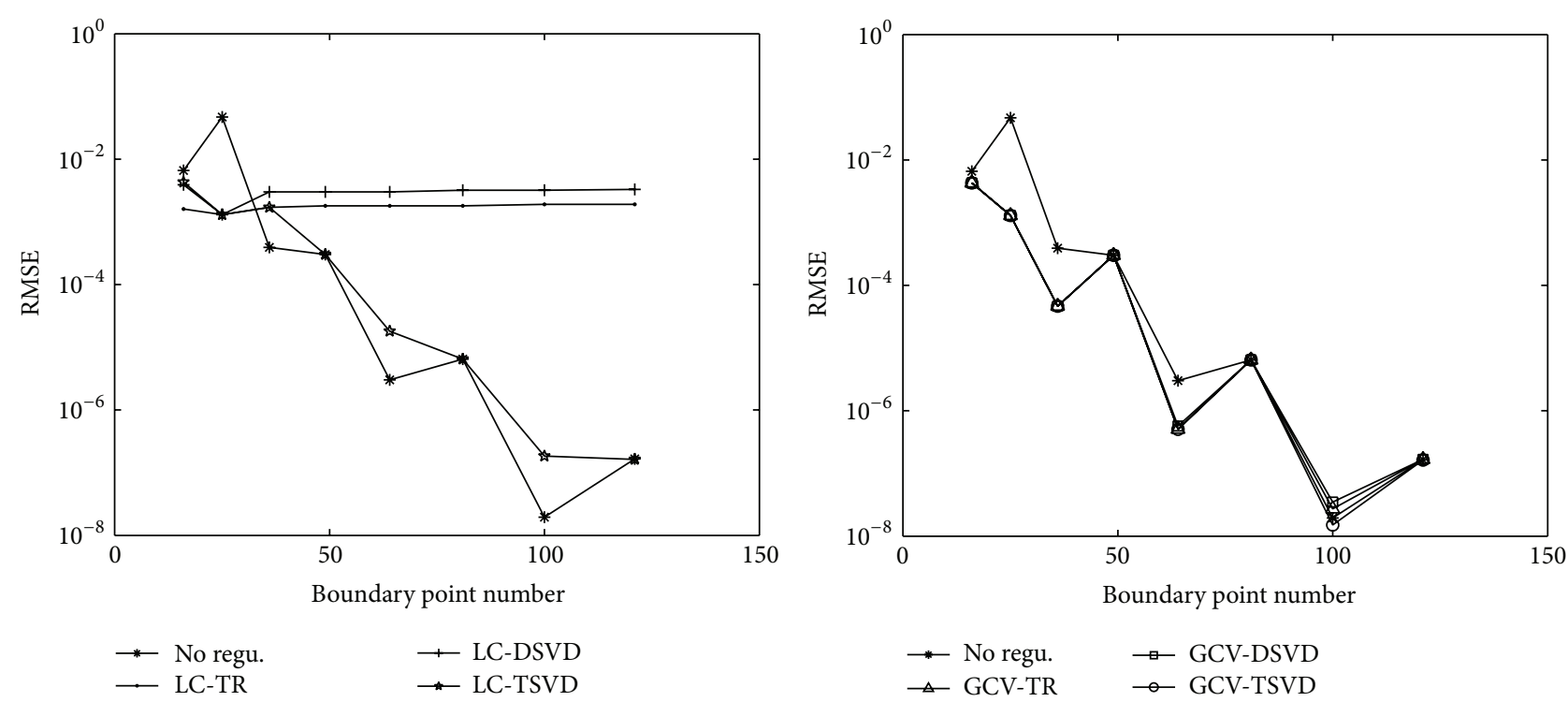

Figure 3: Root mean square errors for case 3.

5.3. Modified Helmholtz Case on a Unit Spherical Domain. In this case, we consider the $3 \mathrm{D}$ modified Helmholtz equation

$$
\Delta u-\lambda^{2} u=0, \quad(x, y, z) \in \Omega
$$

under boundary condition $u=e^{x}+e^{y}+e^{z},(x, y, z) \in \partial \Omega$, with wave number $\lambda=1$.

Figure 3 displays the RMSE versus the boundary point number on a unit spherical domain $\Omega=\left\{(x, y, z) \mid x^{2}+y^{2}+\right.$ $\left.z^{2}<1\right\}$. Similar to case 1 , we find that the convergence curve of the BKM without regularization techniques is oscillatory when the boundary point number becomes large. The result obtained using the LC-DSVD or LC-TR is unacceptable while the LC-TSVD shows a much smoother convergence curve. Even though the GCV based methods have oscillate convergence, they are much better than the one without regularization technique.

For boundary point number $N=100$ with noisy boundary conditions, it is seen from Table 3 that the ECN decreases while the relative average error increases. We observe a sharp drop in the ECN as a tiny amount of noise $\varepsilon=1.0 \times 10^{-5}$ is added. However, the solution is still accurate with $\mathrm{RMSE}=4.61 \times 10^{-4}$. A larger noise $\left(\varepsilon=1.0 \times 10^{-1}\right)$ added leads to unacceptable result RMSE $=4.79 \times 10^{0}$. This shows that this case is similar to case 2 but less sensitive than case 1. By this point, we can believe that the spherical domain is less sensitive than the cubic domain for problems with noisy boundary conditions.

It is noted that the condition number of the cubic domain case is worse than the spherical domain case. We also observe from Figure 4 that the condition number and ECN are larger than those in Figure 2. Similarly, the relation ECN = $O\left(\mathrm{RMSE}^{-1}\right)$ still works here. For different fixed noise levels, we also find that the GCV based regularization techniques give better accuracy than the one with no regularization technique. Different from the cubic domain case, the LCTSVD does not perform well in this case while the other two LC based regularization techniques give better results.

5.4. Modified Helmholtz Case on a Unit Cubic Domain. In this case, we consider the $3 \mathrm{D}$ modified Helmholtz equation

$$
\Delta u-\lambda^{2} u=0, \quad(x, y, z) \in \Omega
$$


TABLE 3: Boundary point number $N=100$ for case 3 .

\begin{tabular}{|c|c|c|c|c|c|}
\hline Noise level & $1 \times 10^{-5}$ & $1 \times 10^{-4}$ & $1 \times 10^{-3}$ & $1 \times 10^{-2}$ & $1 \times 10^{-1}$ \\
\hline Cond & $9.41 \times 10^{19}$ & $9.41 \times 10^{19}$ & $9.41 \times 10^{19}$ & $9.41 \times 10^{19}$ & $9.41 \times 10^{19}$ \\
\hline $\mathrm{ECN}$ & $1.40 \times 10^{5}$ & $1.40 \times 10^{4}$ & $1.40 \times 10^{3}$ & $1.39 \times 10^{2}$ & $1.51 \times 10^{1}$ \\
\hline RMSE & $4.61 \times 10^{-4}$ & $4.80 \times 10^{-3}$ & $4.79 \times 10^{-2}$ & $4.79 \times 10^{-1}$ & $4.79 \times 10^{0}$ \\
\hline LC-TR & $1.90 \times 10^{-3}$ & $1.90 \times 10^{-3}$ & $1.90 \times 10^{-3}$ & $3.00 \times 10^{-3}$ & $5.24 \times 10^{-2}$ \\
\hline LC-DSVD & $3.20 \times 10^{-3}$ & $3.20 \times 10^{-3}$ & $3.40 \times 10^{-3}$ & $7.30 \times 10^{-3}$ & $7.40 \times 10^{-2}$ \\
\hline LC-TSVD & $2.81 \times 10^{-6}$ & $2.50 \times 10^{-5}$ & $2.49 \times 10^{-4}$ & $2.50 \times 10^{-3}$ & $2.49 \times 10^{-2}$ \\
\hline GCV-TR & $2.98 \times 10^{-6}$ & $2.73 \times 10^{-5}$ & $3.57 \times 10^{-4}$ & $2.70 \times 10^{-3}$ & $1.85 \times 10^{-2}$ \\
\hline GCV-DSVD & $3.00 \times 10^{-6}$ & $3.12 \times 10^{-5}$ & $3.22 \times 10^{-4}$ & $2.90 \times 10^{-3}$ & $2.02 \times 10^{-2}$ \\
\hline GCV-TSVD & $2.95 \times 10^{-6}$ & $3.18 \times 10^{-5}$ & $3.65 \times 10^{-4}$ & $2.50 \times 10^{-3}$ & $1.81 \times 10^{-2}$ \\
\hline
\end{tabular}

TABLE 4: Boundary point number $N=488$ for case 4 .

\begin{tabular}{|c|c|c|c|c|c|}
\hline Noise level & 0.0 & $1 \times 10^{-8}$ & $1 \times 10^{-7}$ & $1 \times 10^{-6}$ & $1 \times 10^{-5}$ \\
\hline Cond & $5.06 \times 10^{19}$ & $5.06 \times 10^{19}$ & $5.06 \times 10^{19}$ & $5.06 \times 10^{19}$ & $5.06 \times 10^{19}$ \\
\hline $\mathrm{ECN}$ & $5.55 \times 10^{9}$ & $7.62 \times 10^{7}$ & $7.57 \times 10^{6}$ & $7.56 \times 10^{5}$ & $7.56 \times 10^{4}$ \\
\hline RMSE & $3.27 \times 10^{-4}$ & $7.00 \times 10^{-3}$ & $5.26 \times 10^{-2}$ & $3.53 \times 10^{-1}$ & $9.38 \times 10^{0}$ \\
\hline LC-TR & $2.88 \times 10^{-1}$ & $2.88 \times 10^{-1}$ & $2.88 \times 10^{-1}$ & $1.27 \times 10^{0}$ & $4.66 \times 10^{0}$ \\
\hline LC-DSVD & $1.46 \times 10^{-1}$ & $1.46 \times 10^{-1}$ & $1.46 \times 10^{-1}$ & $6.41 \times 10^{-1}$ & $2.42 \times 10^{0}$ \\
\hline LC-TSVD & $2.00 \times 10^{-3}$ & $1.21 \times 10^{-2}$ & $1.40 \times 10^{-1}$ & $1.42 \times 10^{0}$ & $2.97 \times 10^{-1}$ \\
\hline GCV-TR & $2.90 \times 10^{-3}$ & $1.20 \times 10^{-3}$ & $1.38 \times 10^{-2}$ & $5.53 \times 10^{-2}$ & $6.81 \times 10^{2}$ \\
\hline GCV-DSVD & $6.00 \times 10^{-3}$ & $7.40 \times 10^{-3}$ & $1.97 \times 10^{-2}$ & $5.97 \times 10^{-2}$ & $1.82 \times 10^{-1}$ \\
\hline GCV-TSVD & $1.20 \times 10^{-3}$ & $8.30 \times 10^{-3}$ & $5.70 \times 10^{-3}$ & $6.54 \times 10^{-2}$ & $2.98 \times 10^{-1}$ \\
\hline
\end{tabular}

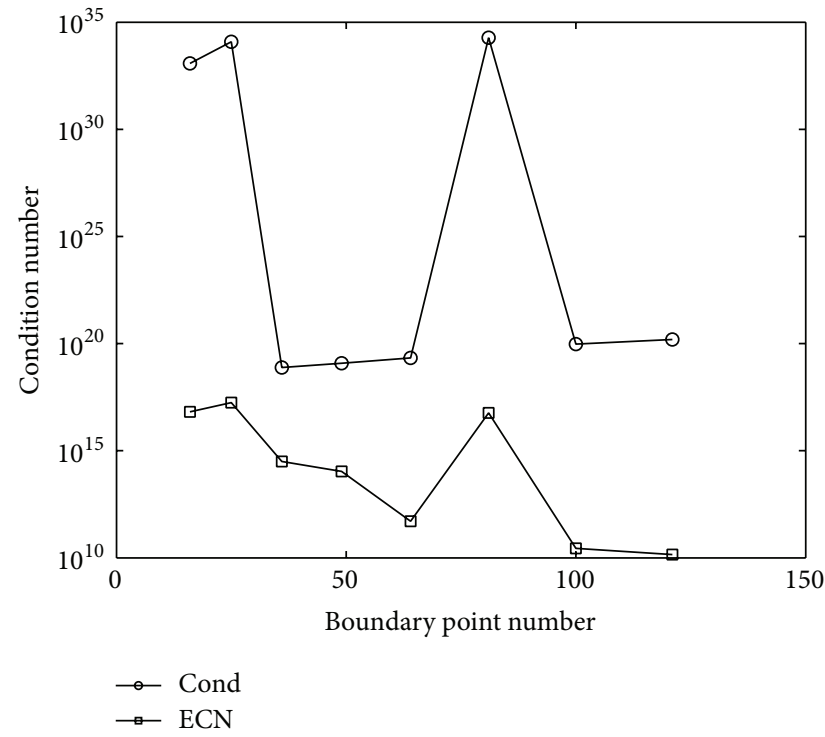

FIGURE 4: Condition number and ECN for case 3.

under Dirichlet boundary condition $u=e^{x+y+z}$ with corresponding wave number $\lambda=\sqrt{3}$.

For fixed boundary point number $N=488$, Table 4 gives the variation of condition number, $\mathrm{ECN}$, and numerical results of the BKM under noisy and noise-free boundary conditions. We find that the boundary point number in this case is larger than case 1, but the numerical solution in case 1 (RMSE $\left.=8.81 \times 10^{-5}\right)$ is better than the one in this case $\left(\right.$ RMSE $\left.=3.27 \times 10^{-4}\right)$. On the other hand, the condition number in case $1\left(\right.$ Cond $\left.=2.39 \times 10^{18}\right)$ is smaller than the one in this case $\left(\right.$ Cond $\left.=5.06 \times 10^{19}\right)$. This phenomenon shows that the character of the Helmholtz equation is better than the modified Helmholtz equation.

Similar to case 1, We observe a sharp drop in the ECN as a tiny amount of noise $\varepsilon=1.0 \times 10^{-5}$ is added. It leads to unacceptable numerical solutions with RMSE $=9.38 \times 10^{\circ}$. For different fixed noise levels, we also find that the GCVDSVD and GCV-TSVD regularization techniques give better accuracy than the one with no regularization technique with about one-decimal precision. Different from the previous three cases, the LC-TSVD performs well in this case while the GCV-TR regularization techniques give unacceptable result for noise $\varepsilon=1.0 \times 10^{-5}$.

\section{Conclusions}

In this paper, the stability analysis of the BKM has been investigated for 3D Helmholtz-type problems. Coupled with three regularization techniques and two algorithms for selecting regularization parameters, we are able to overcome the numerical instability induced from the ill-conditioned BKM interpolation matrix. On the other hand, the effective condition number is introduced to scale the ill-conditioned BKM interpolation matrix.

From the numerical results obtained in the previous section, we observe that the physical domain and boundary value problems have effect on numerical solutions. But this point has no relation to our conclusion; that is, the GCV based regularization techniques perform well for both noisy 
and noise-free boundary conditions. Although the LC based regularization techniques are often used for solving inverse problems with noisy boundary conditions, they are not acceptable for problems tested in this study. It should be noted that the condition of the BKM interpolation system is more dependable on the solution domain for $3 \mathrm{D}$ cases than 2D cases. More interestingly, the highly ill-conditioned interpolation system is less sensitive to the noisy boundary conditions than the less ill-conditioned one.

Besides, numerical results show that the character of the Helmholtz equation is better than the modified Helmholtz equation. Theoretical investigation is under way.

\section{Conflict of Interests}

The authors declare that there is no conflict of interests regarding the publication of this paper.

\section{References}

[1] Y. X. Mukherjee and S. Mukherjee, "The boundary node method for potential problems," International Journal for Numerical Methods in Engineering, vol. 40, no. 5, pp. 797-815, 1997.

[2] M. K. Chati and S. Mukherjee, "The boundary node method for three-dimensional problems in potential theory," International Journal for Numerical Methods in Engineering, vol. 47, no. 9, pp. 1523-1547, 2000.

[3] T. Zhu, J.-D. Zhang, and S. N. Atluri, "A local boundary integral equation (LBIE) method in computational mechanics, and ameshless discretization approach," Computational Mechanics, vol. 21, no. 3, pp. 223-235, 1998.

[4] J. Sladek, V. Sladek, and C. Zhang, "Transient heat conduction analysis in functionally graded materials by the meshless local boundary integral equation method," Computational Materials Science, vol. 28, no. 3-4, pp. 494-504, 2003.

[5] A. Shirzadi, "Meshless local integral equations formulation for the 2D convection-diffusion equations with a nonlocal boundary condition," Computer Modeling in Engineering \& Sciences, vol. 85, no. 1, pp. 45-64, 2012.

[6] E. H. Ooi and V. Popov, "A simplified approach for imposing the boundary conditions in the local boundary integral equation method," Computational Mechanics, vol. 51, no. 5, pp. 717-729, 2013.

[7] J.-M. Zhang, Z.-H. Yao, and H. Li, "A hybrid boundary node method," International Journal for Numerical Methods in Engineering, vol. 53, no. 4, pp. 751-763, 2002.

[8] C. W. Wolgemuth and M. Zajac, "The moving boundary node method: a level set-based, finite volume algorithm with applications to cell motility," Journal of Computational Physics, vol. 229, no. 19, pp. 7287-7308, 2010.

[9] Q. Wang, Y. Miao, and J.-J. Zheng, "The hybrid boundary node method accelerated by fast multipole expansion technique for 3D elasticity," Computer Modeling in Engineering \& Sciences, vol. 70, no. 2, pp. 123-151, 2010.

[10] M. Tatari and F. Ghasemi, "The Galerkin boundary node method for magneto-hydrodynamic (MHD) equation," Journal of Computational Physics, vol. 258, no. 1, pp. 634-649, 2014.

[11] Y. Miao, Q. Wang, H.-P. Zhu, and Y.-P. Li, “Thermal analysis of $3 \mathrm{D}$ composites by a new fast multipole hybrid boundary node method," Computational Mechanics, vol. 53, no. 1, pp. 77-90, 2014.

[12] Y. M. Cheng and M. J. Chen, "A boundary element-free method for elasticity," Acta Mechanica Sinica, vol. 35, no. 2, pp. 181-186, 2003 (Chinese).

[13] Y.-M. Cheng and M.-J. Peng, "Boundary element-free method for elastodynamics," Science in China G, vol. 48, no. 6, pp. 641657, 2005.

[14] K. M. Liew, Y. M. Cheng, and S. Kitipornchai, "Boundary element-free method (BEFM) for two-dimensional elastodynamic analysis using Laplace transform," International Journal for Numerical Methods in Engineering, vol. 64, no. 12, pp. 16101627, 2005.

[15] J.-F. Wang, J.-F. Wang, F.-X. Sun, and Y.-M. Cheng, "An intropolation boundary element-free method with nonsingular weight function for two-dimensional potential problems," International Journal of Computational Methods, vol. 10, no. 6, Article ID 1350043, 23 pages, 2013.

[16] Y.-M. Cheng and M.-J. Peng, "A boundary element-free method (BEFM) for two-dimensional potential problems," Engineering Analysis with Boundary Elements, vol. 33, no. 1, pp. 77-82, 2009.

[17] H.-P. Ren, Y.-M. Cheng, and W. Zhang, "An interpolating boundary element-free method (IBEFM) for elasticity problems," Science in China G, vol. 53, no. 4, pp. 758-766, 2010.

[18] G. Fairweather and A. Karageorghis, "The method of fundamental solutions for elliptic boundary value problems," Advances in Computational Mathematics, vol. 9, no. 1-2, pp. 6995, 1998.

[19] C. S. Chen, A. Karageorghis, and Y. S. Smyrlis, The Method of Fundamental Solutions: A Meshless Method, Dynamic, 2008.

[20] W. Chen and M. Tanaka, "A meshless, integration-free, and boundary-only RBF technique," Computers \& Mathematics with Applications, vol. 43, no. 3-5, pp. 379-391, 2002.

[21] J. T. Chen, M. H. Chang, K. H. Chen, and S. R. Lin, "The boundary collocation method with meshless concept for acoustic eigenanalysis of two-dimensional cavities using radial basis function," Journal of Sound and Vibration, vol. 257, no. 4, pp. 667-711, 2002.

[22] D. L. Young, K. H. Chen, and C. W. Lee, "Novel meshless method for solving the potential problems with arbitrary domain," Journal of Computational Physics, vol. 209, no. 1, pp. 290-321, 2005.

[23] D. L. Young, K. H. Chen, J. T. Chen, and J. H. Kao, "A modified method of fundamental solutions with source on the boundary for solving laplace equations with circular and arbitrary domains," Computer Modeling in Engineering \& Sciences, vol. 19, no. 3, pp. 197-221, 2007.

[24] B. Sarler, "Solution of potential flow problems by the modified method of fundamental solutions: formulations with the single layer and the double layer fundamental solutions," Engineering Analysis with Boundary Elements, vol. 33, no. 12, pp. 1374-1382, 2009.

[25] W. Chen and F. Z. Wang, "A method of fundamental solutions without fictitious boundary," Engineering Analysis with Boundary Elements, vol. 34, no. 5, pp. 530-532, 2010.

[26] Y. J. Liu, "A new boundary meshfree method with distributed sources," Engineering Analysis with Boundary Elements, vol. 34, no. 11, pp. 914-919, 2010.

[27] F. Z. Wang, L. Ling, and W. Chen, "Effective condition number for boundary knot method," Computers, Materials \& Continua, vol. 12, no. 1, pp. 57-70, 2009. 
[28] F. Z. Wang, "Numerical simulation of acoustic problems with highwavenumbers," Applied Mathematics \& Information Sciences. In press.

[29] F.-Z. Wang, W. Chen, and X.-R. Jiang, "Investigation of regularized techniques for boundary knot method," International Journal for Numerical Methods in Biomedical Engineering, vol. 26, no. 12, pp. 1868-1877, 2010.

[30] F. Z. Wang, "Applicability of the boundary particle method," Computer Modeling in Engineering \& Sciences, vol. 80, no. 3-4, pp. 201-217, 2011.

[31] J. Y. Zhang and F. Z. Wang, "Boundary knot method: an overview and some novel approaches," Computer Modeling in Engineering \& Sciences, vol. 88, no. 2, pp. 141-154, 2012.

[32] T. Wei, Y. C. Hon, and L. Ling, "Method of fundamental solutions with regularization techniques for Cauchy problems of elliptic operators," Engineering Analysis with Boundary Elements, vol. 31, no. 4, pp. 373-385, 2007.

[33] F.-Z. Wang, W. Chen, and L. Ling, "Combinations of the method of fundamental solutions for general inverse source identification problems," Applied Mathematics and Computation, vol. 219, no. 3, pp. 1173-1182, 2012.

[34] B.-T. Jin and Y. Zheng, "Boundary knot method for some inverse problems associated with the Helmholtz equation," International Journal for Numerical Methods in Engineering, vol. 62, no. 12, pp. 1636-1651, 2005.

[35] B.-T. Jin and Y. Zheng, "Boundary knot method for the Cauchy problem associated with the inhomogeneous Helmholtz equation," Engineering Analysis with Boundary Elements, vol. 29, no. 10, pp. 925-935, 2005.

[36] P. A. Ramachandran, "Method of fundamental solutions: singular value decomposition analysis," Communications in Numerical Methods in Engineering, vol. 18, no. 11, pp. 789-801, 2002.

[37] C. S. Chen, A. C. Hokwon, and M. A. Golberg, "Some comments on the ill-conditioning of the method of fundamental solutions," Engineering Analysis with Boundary Elements, vol. 30, no. 5, pp. 405-410, 2006.

[38] T. F. Chan and D. E. Foulser, "Effectively well-conditioned linear systems," SIAM Journal on Scientific and Statistical Computing, vol. 9, no. 6, pp. 963-969, 1988.

[39] S. Christiansen and P. C. Hansen, "The effective condition number applied to error analysis of certain boundary collocation methods," Journal of Computational and Applied Mathematics, vol. 54, no. 1, pp. 15-36, 1994.

[40] Z.-C. Li and H.-T. Huang, "Effective condition number for simplified hybrid Trefftz methods," Engineering Analysis with Boundary Elements, vol. 32, no. 9, pp. 757-769, 2008.

[41] T. W. Drombosky, A. L. Meyer, and L. Ling, "Applicability of the method of fundamental solutions," Engineering Analysis with Boundary Elements, vol. 33, no. 5, pp. 637-643, 2009.

[42] J. M. Banoczi, N.-C. Chiu, G. E. Cho, and I. C. F. Ipsen, "The lack of influence of the right-hand side on the accuracy of linear system solution," SIAM Journal on Scientific Computing, vol. 20, no. 1, pp. 203-227, 1998.

[43] P. C. Hansen, "Regularization tools: a Matlab package for analysis and solution of discrete ill-posed problems," Numerical Algorithms, vol. 6, no. 1, pp. 1-35, 1994.

[44] P. C. Hansen, "Analysis of discrete ill-posed problems by means of the L-curve," SIAM Review, vol. 34, no. 4, pp. 561-580, 1992.

[45] Y. C. Hon and T. Wei, "The method of fundamental solution for solving multidimensional inverse heat conduction problems," Computer Modeling in Engineering \& Sciences, vol. 7, no. 2, pp. 119-132, 2005.
[46] J. Lin, W. Chen, and F. Z. Wang, "A new investigation into regularization techniques for the method of fundamental solutions," Mathematics and Computers in Simulation, vol. 81, no. 6, pp. 1144-1152, 2011.

[47] C.-S. Liu, "A modified collocation Trefftz method for the inverse Cauchy problem of Laplace equation," Engineering Analysis with Boundary Elements, vol. 32, no. 9, pp. 778-785, 2008. 


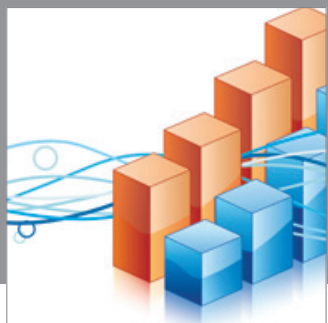

Advances in

Operations Research

mansans

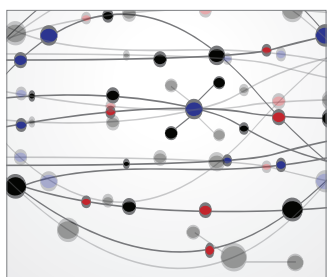

The Scientific World Journal
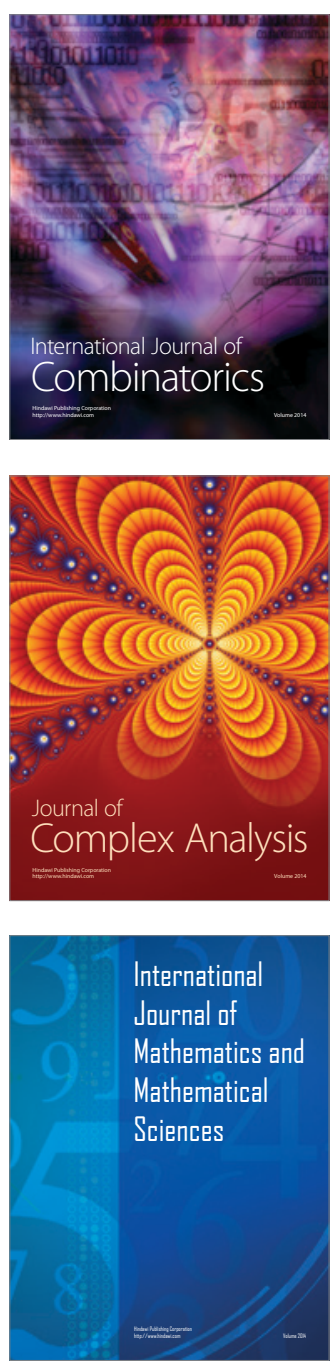
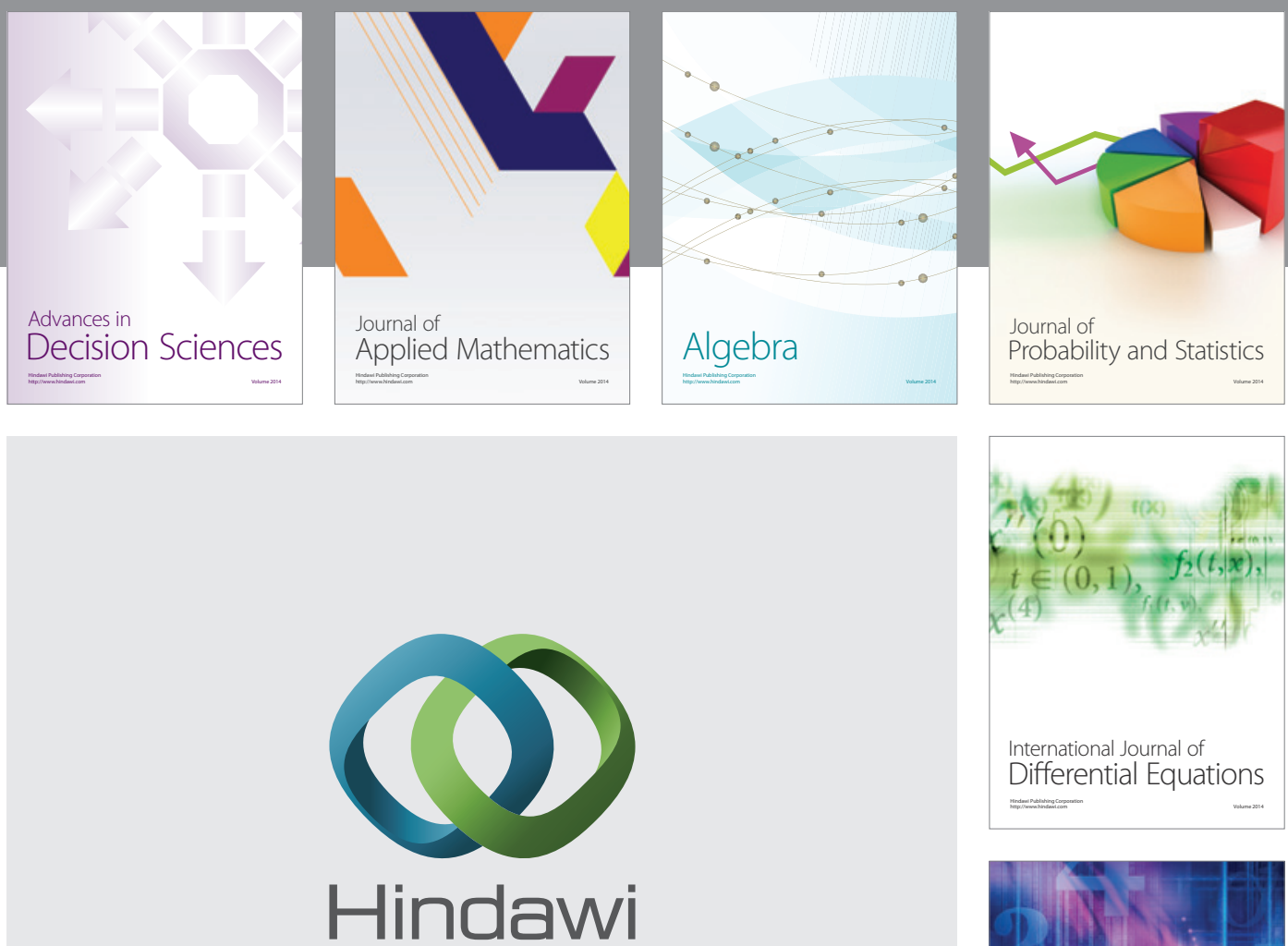

Submit your manuscripts at http://www.hindawi.com
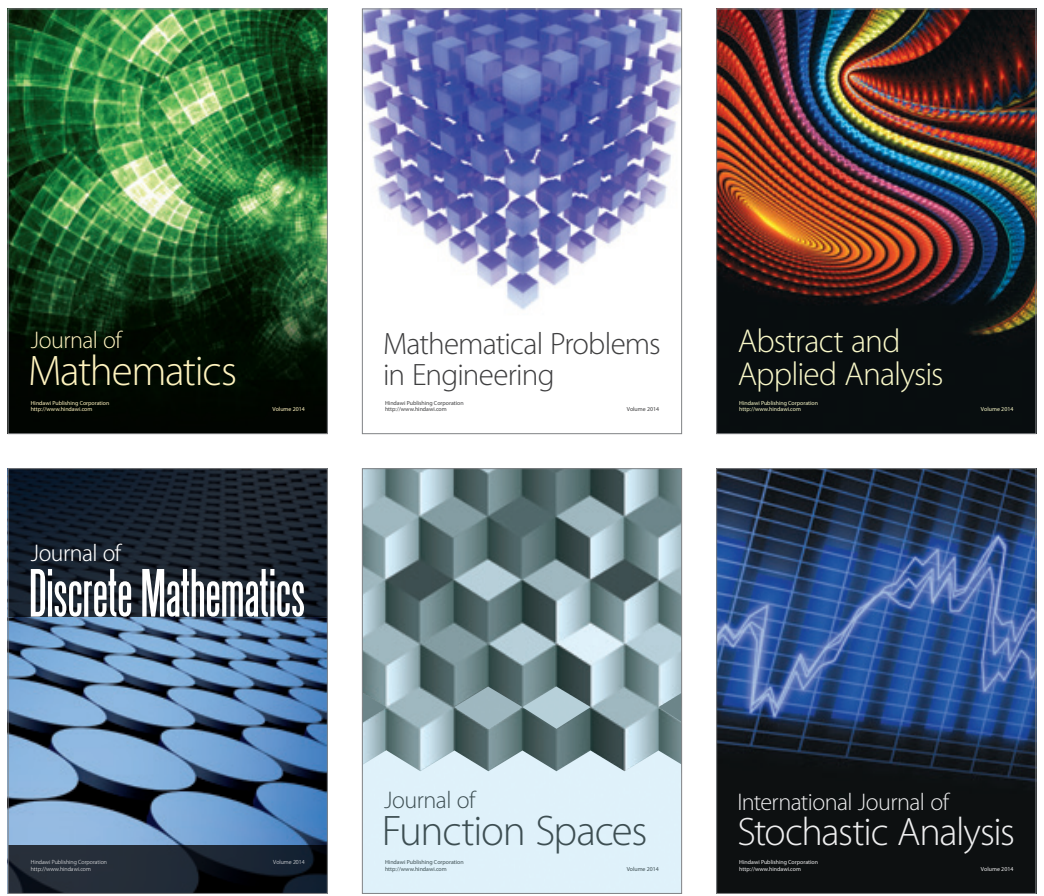

Journal of

Function Spaces

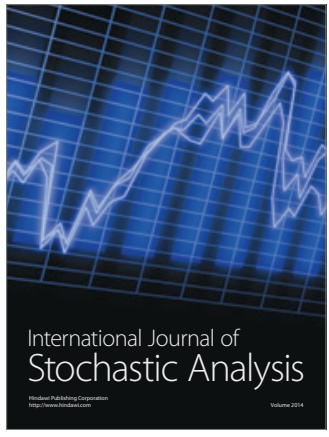

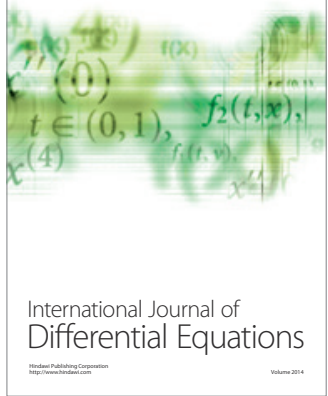
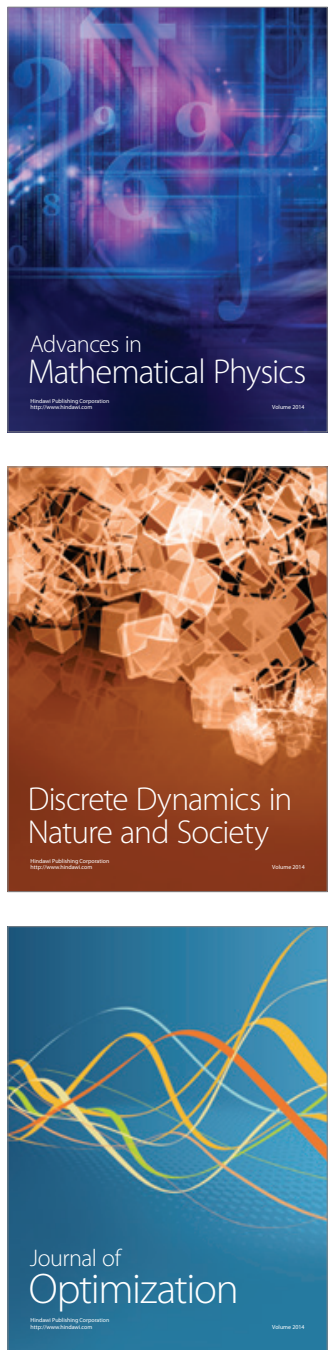